\title{
Machinery Fault Diagnosis Using Two-Channel Analysis Method Based on Fictitious System Frequency Response Function
}

\author{
Kihong Shin ${ }^{1}$ and Sang-Heon Lee ${ }^{2}$ \\ ${ }^{1}$ Department of Mechanical \& Automotive Engineering, Andong National University, 388 Songcheon-Dong, \\ Andong 760-749, Republic of Korea \\ ${ }^{2}$ Department of Mechanical Design, Andong National University, 388 Songcheon-Dong, Andong 760-749, Republic of Korea
}

Correspondence should be addressed to Kihong Shin; kshin@anu.ac.kr

Received 8 January 2015; Revised 7 April 2015; Accepted 17 April 2015

Academic Editor: Roger Serra

Copyright ( $2015 \mathrm{~K}$. Shin and S.-H. Lee. This is an open access article distributed under the Creative Commons Attribution License, which permits unrestricted use, distribution, and reproduction in any medium, provided the original work is properly cited.

\begin{abstract}
Most existing techniques for machinery health monitoring that utilize measured vibration signals usually require measurement points to be as close as possible to the expected fault components of interest. This is particularly important for implementing condition-based maintenance since the incipient fault signal power may be too small to be detected if a sensor is located further away from the fault source. However, a measurement sensor is often not attached to the ideal point due to geometric or environmental restrictions. In such a case, many of the conventional diagnostic techniques may not be successfully applicable. In this paper, a twochannel analysis method is proposed to overcome such difficulty. It uses two vibration signals simultaneously measured at arbitrary points in a machine. The proposed method is described theoretically by introducing a fictitious system frequency response function. It is then verified experimentally for bearing fault detection. The results show that the suggested method may be a good alternative when ideal points for measurement sensors are not readily available.
\end{abstract}

\section{Introduction}

Many diagnostic techniques are based on measured vibration signals for detecting machinery faults. For example, monitoring of variance, kurtosis, power spectrum, and envelope spectrum of a measured vibration signal may be the most common traditional methods to identify any symptom of machinery faults, while the higher order spectral analysis and the time-frequency (or time-scale) analysis have been lately applied for machinery health monitoring. More recently, nonlinear chaotic characteristics such as the correlation dimension, Lyapunov exponent, and Kolmogorov entropy have been examined for the purpose of machinery component health assessment [1], and a fault detection technique based on the principal component analysis (PCA) and the second order blind identification (SOBI) was suggested when a reduced set of sensors is available [2]. Such vibration based diagnostic techniques have become important elements of condition-based maintenance (CBM) in many industrial applications. In particular, various diagnostic and prognostic models for implementing CBM are found in [3]; data fusion strategy is suggested to improve the accuracy of the CBM tool in the process of condition monitoring, diagnostics, and prognostics [4]; the wavelet packet-empirical mode decomposition (WP-EMD) technique is used to extract features for CBM [5]; it is verified that CBM techniques can be used more realistically for making maintenance decisions, and the health of the equipment is determined in situ with the aid of vibration monitoring [6]; an extensive review on diagnostics and prognostics of mechanical systems implementing CBM is given in [7] including the use of multiple sensors.

Several review articles provide general concepts and strategies for using various diagnostic methods based on vibration signals. Specifically, an overview of the principles and techniques of Gaussian time-series methods for fault detection in vibrating structures is given in [8]; some benefits and limitations of time-frequency analysis methods in machinery fault diagnosis are found in [9]; and various fault diagnosis methods for rolling contact bearing are summarized in [10]. 


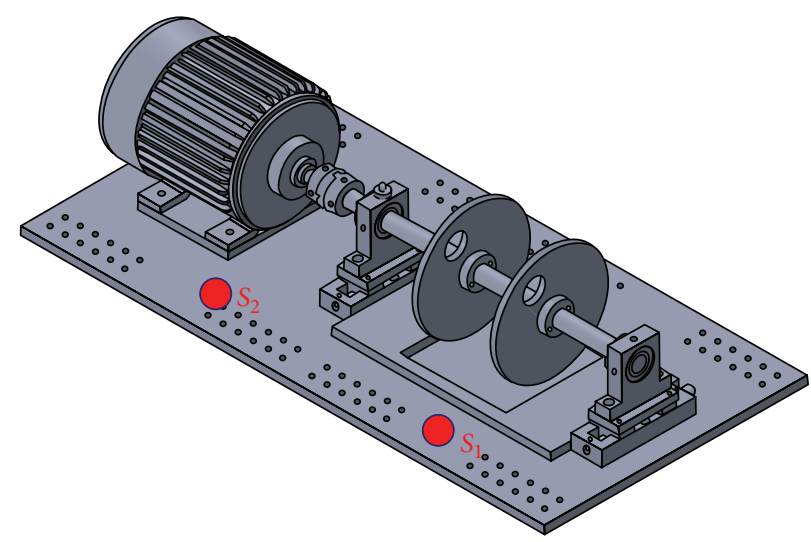

FIGURE 1: Illustrative model of a machine and measurement points $\left(S_{1}\right.$ and $\left.S_{2}\right)$.

Fundamentally, many of vibration based diagnostic techniques used for CBM may be considered as single-channel analysis methods. This is because, even if multichannel signals are measured and used in the analysis, for example, multiple sensors are used to classify fault features using the support vector machine (SVM) [11, 12], the key approach does not considerably differ from the single-channel analysis since the information contained in the observed vibration signals is extracted without explicitly considering the cross relationship between signals. The single-channel analysis method usually requires vibration signals to be measured near the expected fault components of interest to ensure high signal-to-noise ratio of the measured signal. This is very important for implementing condition-based maintenance. In other words, the incipient fault signal power may be too small to be detected if a sensor is located further away from the fault component. Recently, for a large machine with a number of bearing pedestals, the composite spectrum has been introduced to represent the cross relations among all the measured vibration signals $[13,14]$. Similar to the singlechannel analysis method, this method also requires signals measured close to the bearings in order that the composite spectrum properly represents the dynamics of the complete machine assembly.

Even though the use of ideal measurement points is essential for vibration based diagnostic techniques, as is often the case, a measurement sensor might not be attached to the ideal point due to geometric or environmental restrictions (such as when a flat surface for a sensor is not available, the space is too small for the proper attachment of a sensor, the mounting surface is too hot for a general purpose accelerometer, etc.). In such cases, many conventional diagnostic techniques may not be successfully applicable.

In this paper, a two-channel analysis method is proposed for application when ideal points of measurement are not readily available. The proposed method utilizes the crossspectrum between two simultaneously measured vibration signals. A fictitious system frequency response function (FRF) is then constructed [15]. The fictitious FRF compares the cross-spectrums between healthy and faulty conditions and is used to detect any fault symptoms in a machine.
The effectiveness of the proposed method is validated experimentally by applying it to the detection of ball bearing faults. The experimental results show that the proposed method can be a good alternative technique for many machinery health monitoring applications.

\section{Principle of the Two-Channel Analysis Method}

This section describes the principle of the two-channel analysis method. The term "two-channel" is explicitly used because two simultaneously measured signals are required and their cross relations are examined. The details of the proposed method are as follows.

Consider the simple model of a machine as shown in Figure 1, where two measurement points, $S_{1}$ and $S_{2}$, are located far from each other. First, vibration signals are acquired at these points during the healthy operating condition of the machine. Let these two measured vibration signals be $u_{1}(t)$ and $u_{2}(t)$, where each corresponds to the measurement points $S_{1}$ and $S_{2}$, respectively.

If the cross-spectrum between $u_{1}(t)$ and $u_{2}(t)$ is examined, some peaky frequency components might be observed that are correlated with each other, such as the rotor rpm component and the electromagnetically induced motor vibration components. However, since the distance between two measurement points is large, we may assume that two signals are nearly uncorrelated within the frequency range of interest, but not perfectly uncorrelated, such as in the range of the bearing fault frequencies. Thus, for a healthy operating condition, the cross-spectral density function between two signals in this frequency range may be written as

$$
S_{u_{1} u_{2}}(f) \approx 0 \text {. }
$$

Next, suppose there is a new fault in the machine that produces a new excitation force signal $s(t)$, and we obtain vibration signals at the same measurement points. The inputoutput relationship between the excitation source and the two measurement points can then be depicted as shown in Figure 2. Two different paths, $H_{1}$ and $H_{2}$, appear between the source and measurement points. 


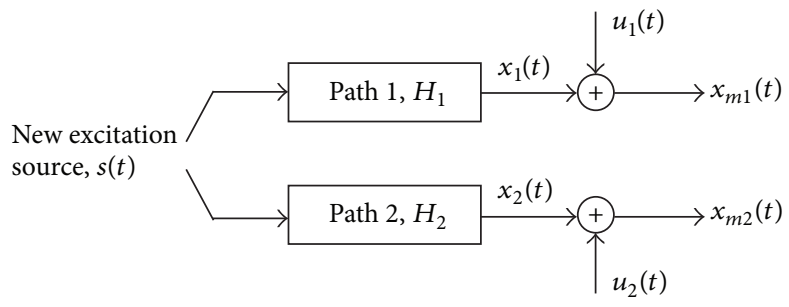

FIGURE 2: Single input-two-output model for a new excitation source.

In Figure 2, signals $x_{1}(t)$ and $x_{2}(t)$ are the components directly related to the fault source, and these are superimposed with the signals corresponding to the healthy operating condition. Thus, the measured signals $x_{m 1}(t)$ and $x_{m 2}(t)$ are

$$
\begin{aligned}
& x_{m 1}(t)=x_{1}(t)+u_{1}(t), \\
& x_{m 2}(t)=x_{2}(t)+u_{2}(t) .
\end{aligned}
$$

If it is assumed that signals $x_{i}(t)$ and $u_{i}(t)$ are uncorrelated with each other within the new fault frequency range, crossspectral density functions between uncorrelated signals are zero; that is, $S_{x_{1} u_{2}}(f)=S_{u_{1} x_{2}}(f)=0$. Since it is also assumed that $S_{u_{1} u_{2}}(f) \approx 0$ in (1), the cross-spectral density function between two measured signals at this particular frequency component can be written as

$$
\begin{aligned}
S_{x_{m 1} x_{m 2}}(f)= & S_{x_{1} x_{2}}(f)+S_{x_{1} u_{2}}(f)+S_{u_{1} x_{2}}(f) \\
& +S_{u_{1} u_{2}}(f) \\
\approx & S_{x_{1} x_{2}}(f)=H_{1}^{*}(f) H_{2}(f) S_{s s}(f),
\end{aligned}
$$

where the symbol “*” denotes the complex conjugate. Equation (3) indicates that the cross-spectrum contains both the fault source power and the path relations. This crossspectrum may be directly used to detect any new fault. However, as mentioned earlier, the fault source power contained in the cross-spectral density function may be too small to observe properly because the measurement sensors are not close to the ideal points near the fault source. This problem may be overcome by using the concept of the fictitious system frequency response function that is used to relate two deterministic signals [15].

2.1. Construction of the Fictitious System Frequency Response. We now describe the details of building the fictitious system frequency response function as follows. It is noted that, even if the above measured vibration signals may be random, especially within the frequency range of interest, the estimated cross-spectrums may be classified as deterministic for a particular operating condition. Then, although there is no physical input-output relationship between the two crossspectral density functions, $S_{u_{1} u_{2}}(f)$ and $S_{x_{m 1} x_{m 2}}(f)$, it can be possible to relate them artificially, as shown in Figure 3.

The fictitious frequency response function, $H_{\text {fic }}(f)$, informs us of the changes in the machine with respect to the healthy operating condition. The fictitious frequency

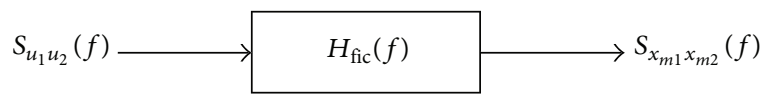

FIgURE 3: Fictitious linear time-invariant system.

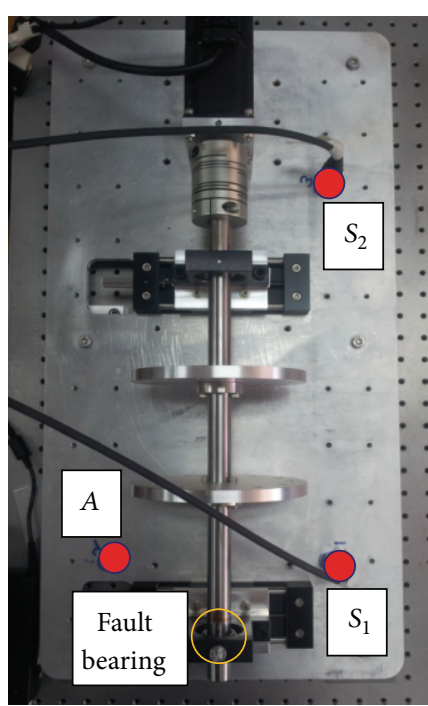

Figure 4: Experimental setup and measurement points $\left(S_{1}\right.$ and $S_{2}$ are the main measurement points, and $A$ is an auxiliary measurement point).

response function can then be examined in terms of both the magnitude and the phase as follows:

$$
H_{\mathrm{fic}}(f)=\frac{H_{1}^{*}(f) H_{2}(f) S_{s s}(f)}{S_{u_{1} u_{2}}(f)}=\left|H_{\mathrm{fic}}(f)\right| e^{j \phi_{\mathrm{fic}}(f)}
$$

where $\left|H_{\text {fic }}(f)\right|$ and $\phi_{\text {fic }}(f)$ are the magnitude and phase of the fictitious system frequency response function and the symbol " $j$ " denotes the imaginary unit $j=\sqrt{-1}$. This fictitious frequency response function has a significant advantage in its ability to detect a new fault power over the power spectrum or the cross-spectrum. Note that the values of the cross-spectral density function obtained in a healthy operating condition are very small in the frequency range of interest. That is, the denominator of (4) is very small. Thus, if a new fault signal arises within this frequency range, the fictitious frequency response function can easily increase the fault frequency component by magnifying the fault source power, even if the source power contained in the measured signal is weak. 


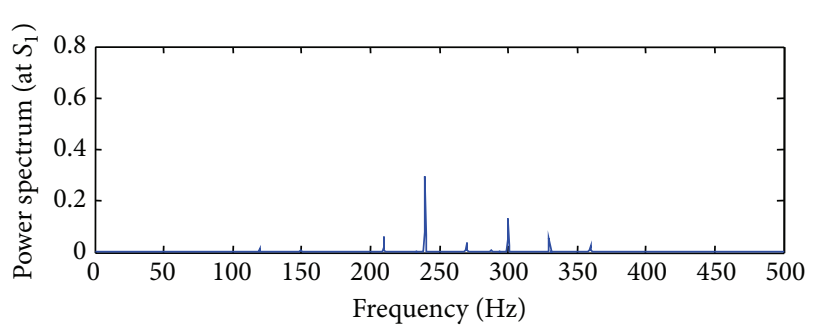

(a)

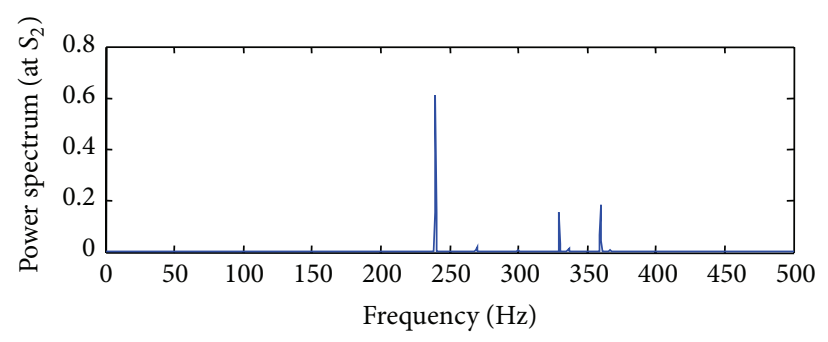

(b)

Figure 5: Power spectrums in a healthy condition: (a) measured at $S_{1}$ and (b) measured at $S_{2}$.

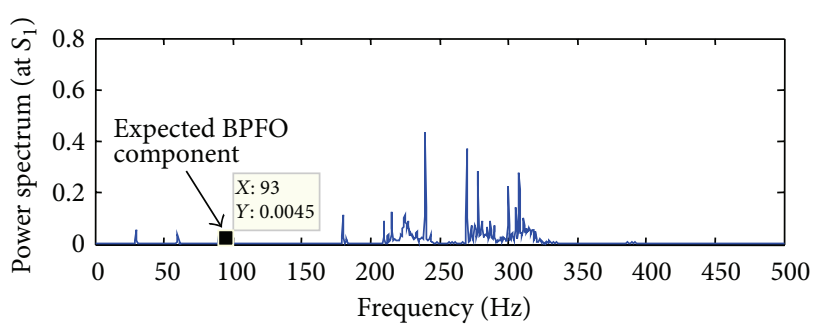

(a)

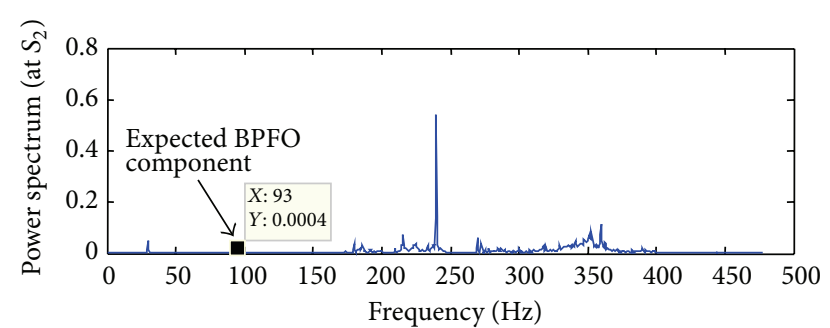

(b)

FIgURE 6: Power spectrums in the case of a fault on the outer race of the ball bearing: (a) measured at $S_{1}$ and (b) measured at $S_{2}$.

Another benefit of using the fictitious frequency response function is that, because the two paths, $H_{1}$ and $H_{2}$, have the phase components, the fault symptom must be reflected in the phase component. Therefore, the phase components of $H_{\text {fic }}(f)$ can also be used to further verify the diagnostic results by monitoring any phase change near the fault frequency component. This may enhance the diagnostic results.

\section{Experimental Results}

The proposed method is applied to detect ball bearing faults using a specially designed fault simulator as shown in Figure 4. The shaft rotates at $1800 \mathrm{rpm}(30 \mathrm{~Hz})$ and two measurement accelerometers are located at some distance from both the motor-end bearing and the far-end bearing. Two artificial defects are considered in the far-end bearing: a fault on the outer race of the bearing and a fault on the inner race. The corresponding bearing fault frequencies are given below [16]:

BPFO (Ball-pass frequency, outer race)

$$
=\frac{n f_{r}}{2}\left(1-\frac{d}{D} \cos \phi\right)
$$

BPFI (Ball-pass frequency, inner race)

$$
=\frac{n f_{r}}{2}\left(1+\frac{d}{D} \cos \phi\right)
$$

where $f_{r}$ is the shaft's rotating frequency, $n$ is the number of balls, $d$ is the ball diameter, $D$ is the pitch diameter of the bearing, and $\phi$ is the angle of the load from the radial plane. The parameters of the ball bearing used in this experiment are $n=8, d \approx 7.9, D=34.5$, and $\phi=0$. Thus, for the shaft speed of $30 \mathrm{~Hz}$, the corresponding BPFO and BPFI components are expected to be about $93 \mathrm{~Hz}$ and $147 \mathrm{~Hz}$, respectively.

First, the power spectrums of the three cases (healthy, outer race fault, and inner race fault) are examined. Figure 5 shows the power spectrums measured at both measurement points, $S_{1}$ and $S_{2}$, in a healthy operating condition. They show that the dominant frequencies are the electromagnetically induced motor vibration component at $240 \mathrm{~Hz}$ and some unknown higher frequency components.

Figures 6 and 7 show the power spectrums in the cases of the outer race fault and the inner race fault, respectively. For both cases, some notable changes are observed in the power spectrums compared with the case of healthy operating condition. However, the expected bearing fault frequency components around $93 \mathrm{~Hz}$ for the outer race fault and around $147 \mathrm{~Hz}$ for the inner race fault are barely visible, while the dominant frequency component of $240 \mathrm{~Hz}$ remains the same. This is mainly because signals are not measured close to the fault source.

The fictitious frequency response functions are then examined for both cases of bearing fault. For the outer race fault, as shown in Figure 8(a), the magnitude spectrum of the fictitious FRF distinctively reveals the BPFO component of $93 \mathrm{~Hz}$. It is noted that any other frequency components are not visible clearly while the BPFO component is greatly magnified. This is another benefit of using the proposed method; the fictitious FRF tends to significantly magnify a new fault component while the existing frequency components are relatively scaled down. It is also noted that the phase spectrum in Figure 8(b) shows the abrupt change of the phase component around $93 \mathrm{~Hz}$. This further verifies the BPFO component. 


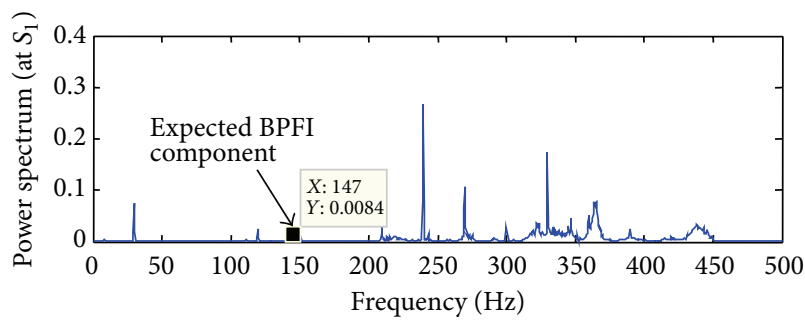

(a)

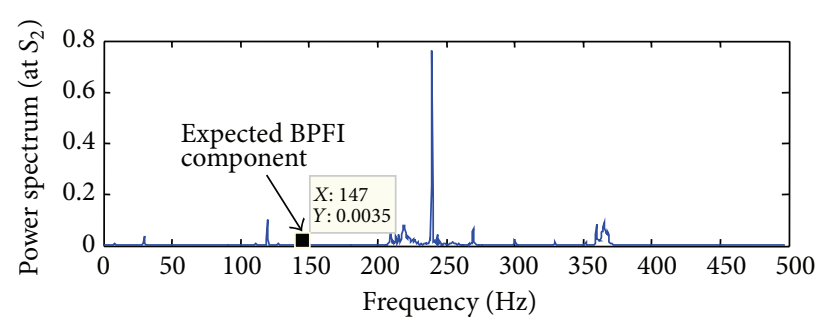

(b)

FIGURE 7: Power spectrums in the case of a fault on the inner race of the ball bearing: (a) measured at $S_{1}$ and (b) measured at $S_{2}$.

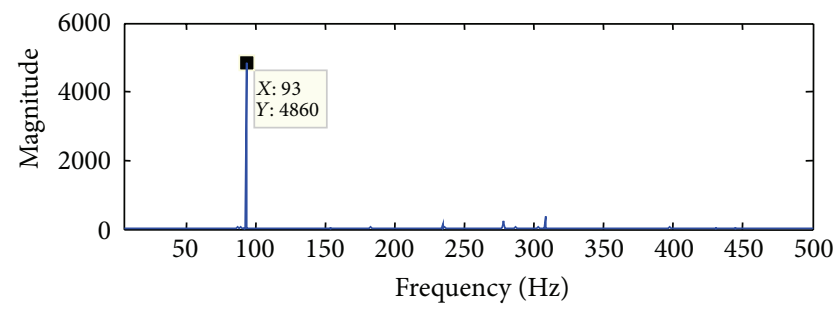

(a)

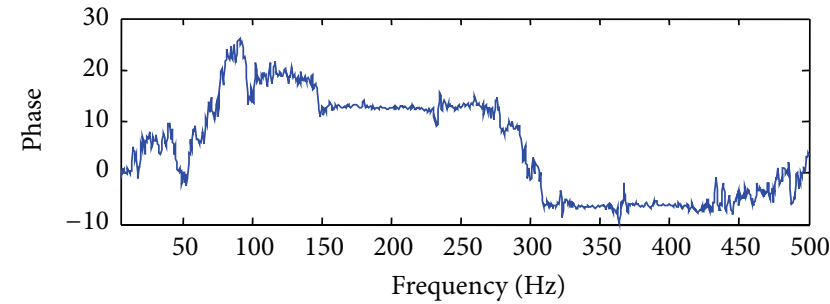

(b)

FIGURE 8: Fictitious frequency response function in the case of a fault on the outer race of the ball bearing: (a) magnitude spectrum and (b) phase spectrum.

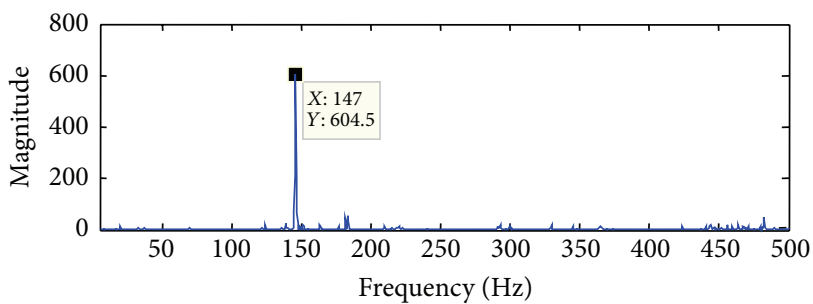

(a)

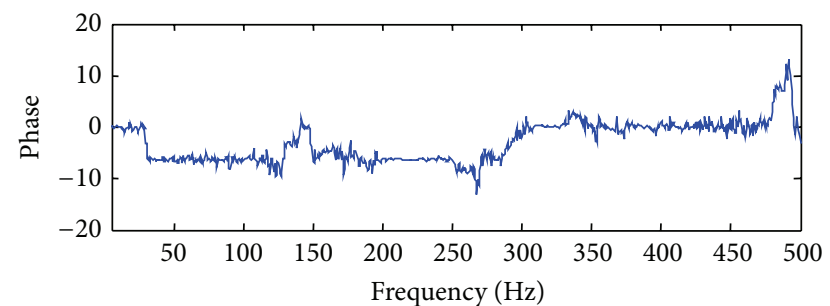

(b)

FIGURE 9: Fictitious frequency response function in the case of a fault on the inner race of the ball bearing: (a) magnitude spectrum and (b) phase spectrum.

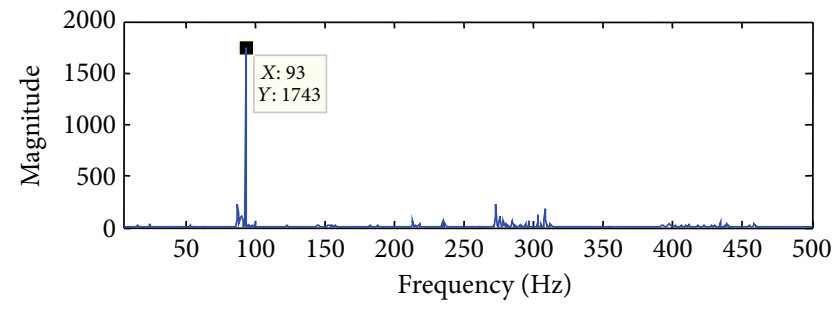

(a)

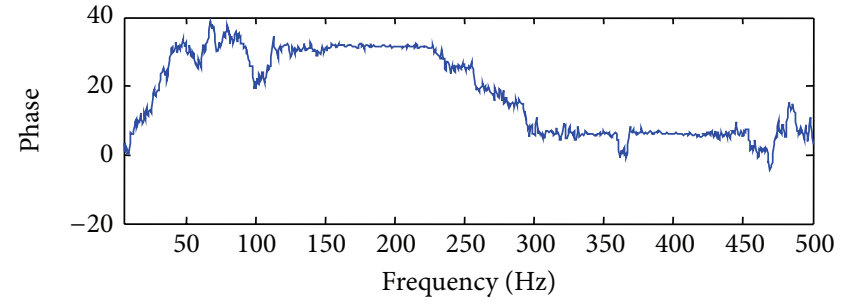

(b)

FIGURE 10: Fictitious frequency response function in the case of a fault on the outer race of the ball bearing (sensor at $S_{1}$ is moved to the point $A$ in Figure 4): (a) magnitude spectrum and (b) phase spectrum.

Figure 9 shows similar results for the case of the inner race fault. That is, the magnitude spectrum of the fictitious FRF significantly magnifies the BPFI component of $147 \mathrm{~Hz}$, and the phase spectrum shows the abrupt change of phase around the BPFI component.
To further verify the method, the sensor at the point $S_{1}$ is moved to the point $A$ in Figure 4. The results are shown in Figures 10 and 11 for the cases of the outer race fault and the inner race fault, respectively. As before, it is found that the magnitude spectrum of the fictitious FRF highlights the 


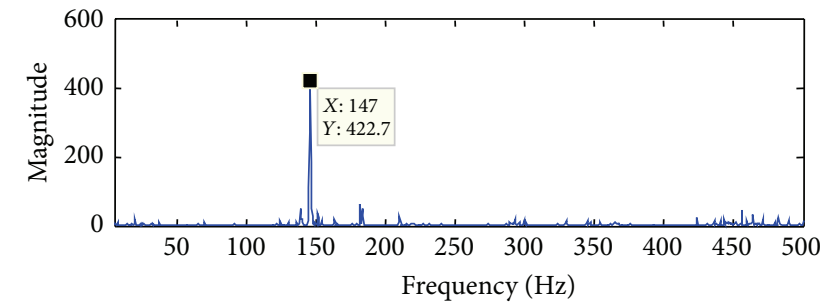

(a)

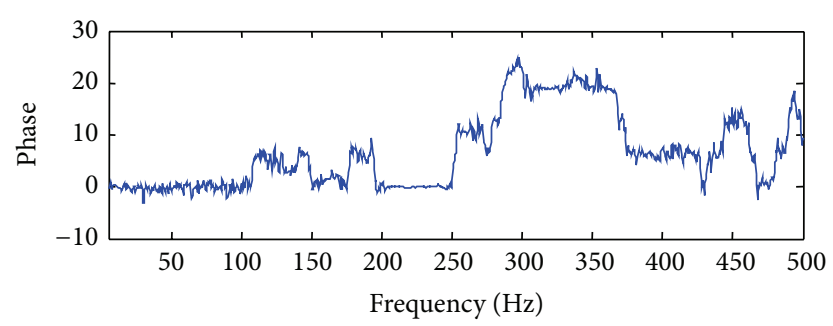

(b)

FIGURE 11: Fictitious frequency response function in the case of a fault on the inner race of the ball bearing (sensor at $S_{1}$ is moved to the point $A$ in Figure 4): (a) magnitude spectrum and (b) phase spectrum.

fault component only, and a sudden change of phase near the fault component is observed in the phase spectrum. These results may ensure that the suggested method is not sensitive to the measurement points as long as the distance between two measurement points is sufficiently large.

\section{Conclusions}

In this paper, a new strategy for machinery health monitoring is introduced by using two simultaneously measured vibration signals. The locations of measurement sensors are not important; almost arbitrary points may be selected provided the distance between the two measurement points is sufficient. The key process involves the use of the fictitious frequency response function as the means of comparing the cross-spectral density functions between healthy and faulty operating conditions.

Experimental results demonstrate that the proposed method can be a good alternative technique, especially when measurement points need to be flexible. Particularly, the method utilizes both the magnitude spectrum and the phase spectrum of the fictitious frequency response function and can thus produce a more reliable diagnosis result.

\section{Conflict of Interests}

The authors declare that there is no conflict of interests regarding the publication of this paper.

\section{Acknowledgments}

This work was supported by Daejoo Machinery, Andong National University Cooperative Research Center, and Daejoo Machinery Co. Ltd., Daegu, Republic of Korea.

\section{References}

[1] C. Lu, Q. Sun, L. Tao, H. Liu, and C. Lu, "Bearing health assessment based on chaotic characteristics," Shock and Vibration, vol. 20, no. 3, pp. 519-530, 2013.

[2] V. H. Nguyen, C. Rutten, and J.-C. Golinval, "Fault diagnosis in industrial systems based on blind source separation techniques using one single vibration sensor," Shock and Vibration, vol. 19, no. 5, pp. 795-801, 2012.
[3] Y. Peng, M. Dong, and M. J. Zuo, "Current status of machine prognostics in condition-based maintenance: a review," The International Journal of Advanced Manufacturing Technology, vol. 50, no. 1-4, pp. 297-313, 2010.

[4] G. Niu, B.-S. Yang, and M. Pecht, "Development of an optimized condition-based maintenance system by data fusion and reliability-centered maintenance," Reliability Engineering \& System Safety, vol. 95, no. 7, pp. 786-796, 2010.

[5] S. Hong, Z. Zhou, E. Zio, and K. Hong, "Condition assessment for the performance degradation of bearing based on a combinatorial feature extraction method," Digital Signal Processing, vol. 27, no. 1, pp. 159-166, 2014.

[6] R. Ahmad and S. Kamaruddin, "An overview of time-based and condition-based maintenance in industrial application," Computers and Industrial Engineering, vol. 63, no. 1, pp. 135-149, 2012.

[7] A. K. S. Jardine, D. Lin, and D. Banjevic, "A review on machinery diagnostics and prognostics implementing condition-based maintenance," Mechanical Systems and Signal Processing, vol. 20, no. 7, pp. 1483-1510, 2006.

[8] S. D. Fassois and J. S. Sakellariou, "Time-series methods for fault detection and identification in vibrating structures," Philosophical Transactions of the Royal Society of London. Series A. Mathematical, Physical and Engineering Sciences, vol. 365, no. 1851, pp. 411-448, 2007.

[9] Z. Feng, M. Liang, and F. Chu, "Recent advances in timefrequency analysis methods for machinery fault diagnosis: a review with application examples," Mechanical Systems and Signal Processing, vol. 38, no. 1, pp. 165-205, 2013.

[10] P. Jayaswal, A. K. Wadhwani, and K. B. Mulchandani, "Machine fault signature analysis," International Journal of Rotating Machinery, vol. 2008, Article ID 583982, 10 pages, 2008.

[11] L.-L. Jiang, H.-K. Yin, X.-J. Li, and S.-W. Tang, "Fault diagnosis of rotating machinery based on multisensor information fusion using SVM and time-domain features," Shock and Vibration, vol. 2014, Article ID 418178, 8 pages, 2014.

[12] C.-W. Fei, G.-C. Bai, W.-Z. Tang, and S. Ma, "Quantitative diagnosis of rotor vibration fault using process power spectrum entropy and support vector machine method," Shock and Vibration, vol. 2014, Article ID 957531, 9 pages, 2014.

[13] J. K. Sinha and K. Elbhbah, "A future possibility of vibration based condition monitoring of rotating machines," Mechanical Systems and Signal Processing, vol. 34, no. 1-2, pp. 231-240, 2013.

[14] K. Elbhbah and J. K. Sinha, "Vibration-based condition monitoring of rotating machines using a machine composite spectrum," Journal of Sound and Vibration, vol. 332, no. 11, pp. 2831$2845,2013$. 
[15] K. Shin, H. Yang, S.-K. Lee, and Y.-S. Lee, "Group delay based location template matching method for the identification of the impact location on a plate," Journal of Sound and Vibration, vol. 332, no. 8, pp. 2111-2117, 2013.

[16] R. B. Randall and J. Antoni, "Rolling element bearing diagnostics-a tutorial," Mechanical Systems and Signal Processing, vol. 25, no. 2, pp. 485-520, 2011. 

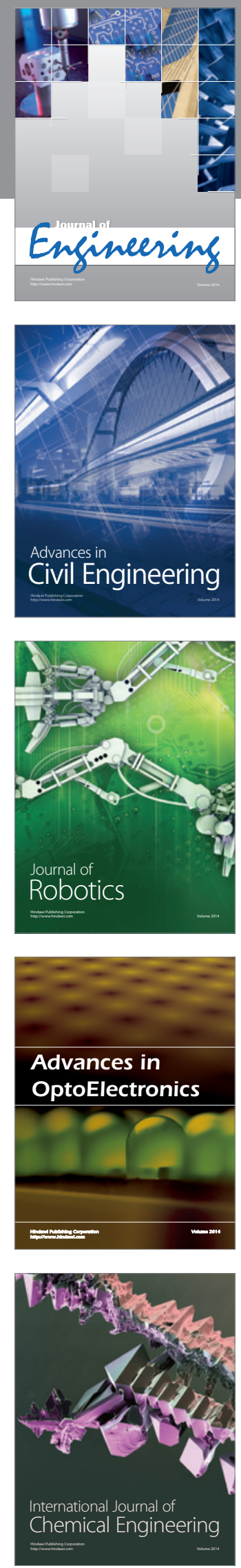

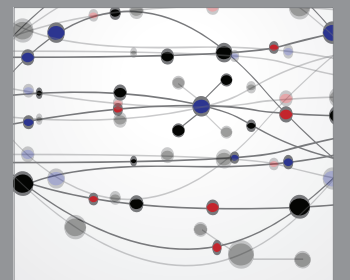

The Scientific World Journal
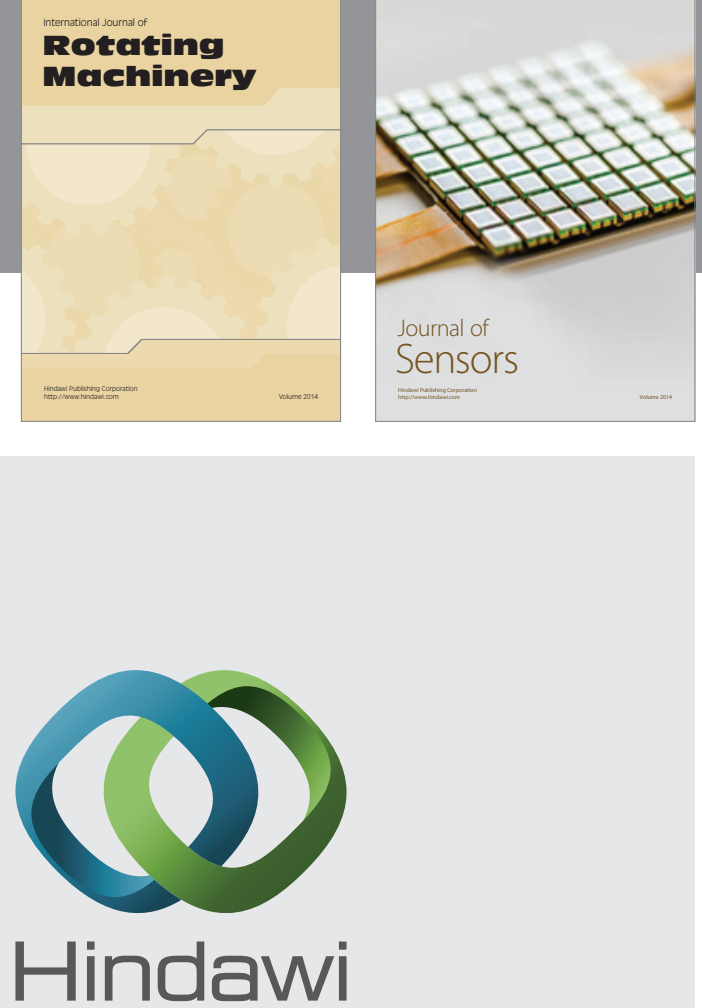

Submit your manuscripts at http://www.hindawi.com
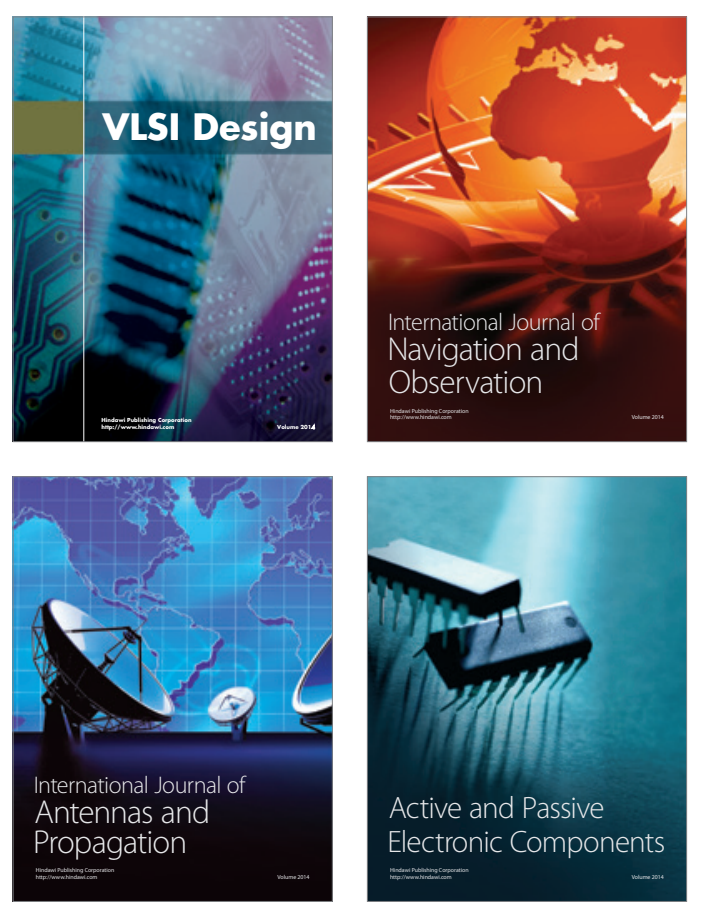
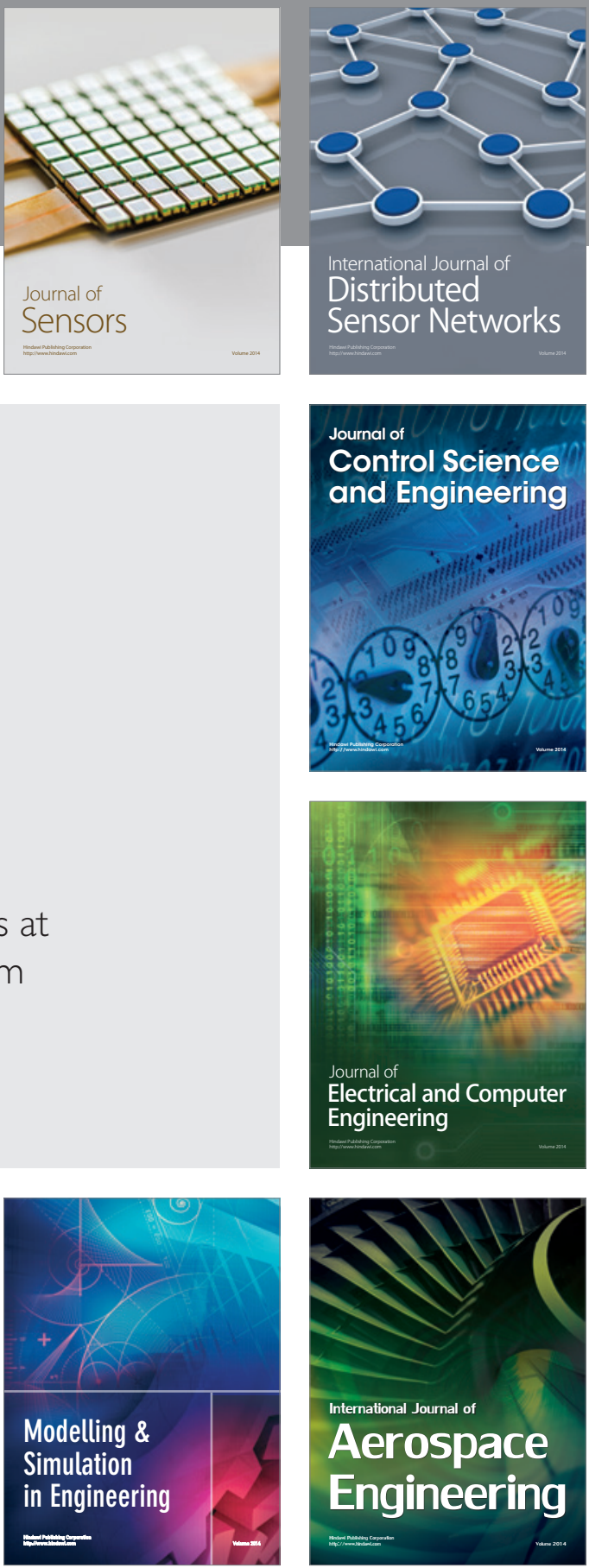

Journal of

Control Science

and Engineering
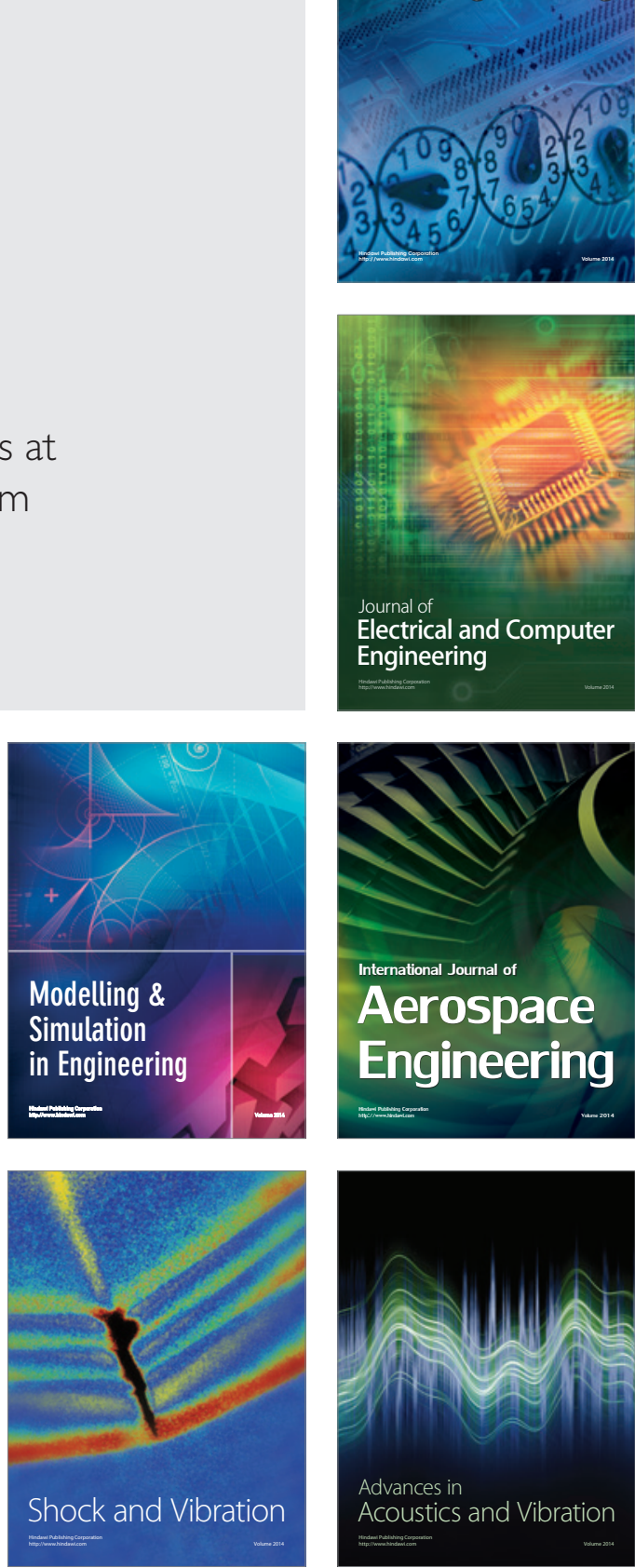